\title{
Nonlinear Inversion in TE Scattering
}

\author{
Bert Jan Kooij and Peter M. van den Berg
}

\begin{abstract}
A method for reconstructing the complex permittivity of a bounded inhomogeneous object from measured scatteredfield data is presented. This paper extends the method previously developed for the TM case to the more complicated TE case. In the TM case, the electric-field integral equation involves an integral operator whose integrand was simply a product of the background Green's function, contrast, and field. In the TE case, the magnetic field is polarized along the axis of an inhomogeneous cylinder of arbitrary cross section and the corresponding integral equation contains derivatives of both the background Green's function and the field. The nonlinear inversion based upon the modified-gradient method as presented in the literature is applied to the magnetic-field equation. However, the integral equation can also be formulated as an electric-field integral equation for the two transversal components of the electric field. Again, the integrand is a product of the background Green's function, contrast, and electric-field vector. The derivatives are operative outside the integral. In this paper, the latter formulation will be taken as a point of departure to develop a nonlinear inversion scheme using the modified-gradient method.
\end{abstract}

Index Terms-Electromagnetic waves, nonlinear inversion.

\section{INTRODUCTION}

$\mathbf{I}$ $\mathrm{N}$ THE reconstruction of material shapes and properties, we often distinguish between two different classes of objects. One class involves the determination of the constitutive parameters of a penetrable scatterer (permittivity, permeability, and conductivity), while the second class is concerned with determining the shape of the boundary of an impenetrable scatterer. In both classes, the orientation and location of the scatterer are also of interest. From the illumination of the scatterer by known sources and measurement of the field at distinct locations exterior to the object, together with specific reconstruction algorithms and a priori information, a reconstruction of the scatterer can be obtained. The use of the a priori information, whether the scatterer is penetrable or impenetrable, is standard in designing reconstruction algorithms.

In this paper, both classes of scatterers are investigated in case the scatterer is illuminated by TE-polarized source fields. This paper is an extension of the method described by Kleinman and Van den Berg [1]-[3] to the case of illumination by TE-polarized source fields. The method is based on casting the inverse problem as an optimization problem in which the cost functional is the sum of two terms: one is the defect in matching measured (actual or synthetic) field data with the field scattered by a body with a particular permittivity,

Manuscript received September 29, 1997; revised April 15, 1998.

The authors are with the Centre for Technical Geoscience, Laboratory of Electromagnetic Research, Department of Electrical Engineering, Delft University of Technology, $2600 \mathrm{GA}$ Delft, The Netherlands.

Publisher Item Identifier S 0018-9480(98)08296-9. permeability, and conductivity, and the second is the error in satisfying the equations of state integral equations for the field produced in the body by each excitation of the known sources. The index and fields are each updated by a linear iterative method in which the updating directions are weighted by parameters which are determined by minimizing the cost functional.

In the TE case, the domain integral equation can be formulated as a scalar-domain integral equation with the one nonzero magnetic-field component as the unknown field [4], [5] or in terms of a vector integral equation with the electric-field vector (two nonzero components) as the unknown field. It is shown in this paper that the single magnetic-field equation turns out to be inferior to the dual electric-field equation. The magneticfield equation is not as stable and not as numerically efficient as the electric-field equation. The electric-field equation further has the feature that the formulation can easily be extended to the three-dimensional case (one additional equation of the same type).

The main distinction between this paper and earlier work done by Kleinman and Van den Berg is that in the case of TE-polarized illumination, the domain integral equation which occurs covers the object domain and contains a gradientdivergence operator. It acts on the vector potential (the vector potential is the convolution of the free-space Green function and contrast source). In order to solve this singular integral equation numerically, we have to take care that in our numerical scheme no artificial surface currents (Dirac delta functions in the domain equation) are generated. One can achieve this by considering that the spatial derivatives that occur in the integral equation should only act on a spatially continuous function. By imposing this condition on our numerical discretization scheme of the integral equation, we developed a scheme that was quite similar to the one developed by Zwamborn and Van den Berg [6]. First, we perform an expansion of the vector potential in which the nature of the expansion depends on the operator that is acting on it. In the discretization of the divergence operator that acts on the vector potential, we use a spatially continuous expansion function, like the rooftop function, in the direction of the spatial derivative. By introducing a suitable weighting procedure, we have the opportunity to let spatial derivatives that occur in the gradient operator act on the weighting function. Similar as for the expansion function, we use for the weighting function a spatially continuous function, like the rooftop function, in the direction of the spatial derivative. This procedure is also applicable to the discretization of the three-dimensional-domain integral equation with contrast in the permittivity, as well as contrast in the permeability. 


\section{INTEGRAL REPRESENTATIONS}

Consider the scattering object to be an inhomogeneous lossy dielectric cylinder of arbitrary cross section $\mathrm{D}$ imbedded in free space. The incident field consists of electromagnetic waves with the magnetic vector polarized along the cylinder axis. To reconstruct the complex permittivity of an unknown object from a knowledge of the scattered field, we assume that the object is illuminated successively by a number of different excitations. For each excitation [time factor is $\exp (-i \omega t)$ ], we assume that the scattered magnetic field is measured exterior to the scatterer. The vectorial position in the two-dimensional space is denoted by $\boldsymbol{x}=\left(x_{1}, x_{2}\right)$. The complex permittivity of the inhomogeneous dielectric object is given by

$$
\varepsilon(\boldsymbol{x})=\varepsilon_{r} \varepsilon_{0}+i \sigma / \omega
$$

where $\varepsilon_{r}$ denotes the relative permittivity of the object with respect to the lossless and homogeneous embedding with permittivity $\varepsilon_{0}$, and $\sigma$ denotes the electric conductivity of the object. The incident electric-field vector is denoted as $\boldsymbol{E}^{\text {inc }}=\left(E_{1}^{\text {inc }}, E_{2}^{\text {inc }}\right)$ and the incident magnetic-field vector $\boldsymbol{H}^{\text {inc }}$ has only the component $H_{3}^{\text {inc }}$. When we assume that the magnetic-field vector is given, the electric-field vector $\boldsymbol{E}^{\text {inc }}=$ $\left(E_{1}^{\text {inc }}, E_{2}^{\text {inc }}\right)$ follows from $E^{\text {inc }}=\left(-i \omega \varepsilon_{0}\right)^{-1} \nabla \times H^{\text {inc }}$ or

$$
\begin{aligned}
& E_{1}^{\text {inc }}=-\left(i \omega \varepsilon_{0}\right)^{-1} \partial_{2} H_{3}^{\text {inc }} \\
& E_{2}^{\text {inc }}=\left(i \omega \varepsilon_{0}\right)^{-1} \partial_{1} H_{3}^{\text {inc }} .
\end{aligned}
$$

The electromagnetic field scattered by the two-dimensional object is obtained as

$$
\boldsymbol{E}^{\mathrm{sct}}=\boldsymbol{E}-\boldsymbol{E}^{\mathrm{inc}} \quad H_{3}^{\mathrm{sct}}=H_{3}-H_{3}^{\mathrm{inc}}
$$

where the $\boldsymbol{E}=\left(E_{1}, E_{2}\right)$ and $H_{3}$ denotes the total electromagnetic field present in the configuration. For the scattered field, we have the integral representations in terms of contrast sources, viz.,

$$
\begin{array}{r}
\boldsymbol{E}^{\mathrm{sct}}(\boldsymbol{x})=\left(k_{0}^{2}+\nabla \nabla \cdot\right) \int_{\boldsymbol{x}^{\prime} \in \mathbf{D}} \frac{i}{4} H_{0}^{(1)}\left(k_{0}\left|\boldsymbol{x}-\boldsymbol{x}^{\prime}\right|\right) \\
\cdot \chi\left(\boldsymbol{x}^{\prime}\right) \boldsymbol{E}\left(\boldsymbol{x}^{\prime}\right) d v
\end{array}
$$

where $\mathbf{D}$ denotes the contrasting domain, $k_{0}=\omega\left(\varepsilon_{0} \mu_{0}\right)^{1 / 2}$ is the wavenumber, and where the normalized material contrast is given by

$$
\chi(\boldsymbol{x})=\frac{\varepsilon(\boldsymbol{x})-\varepsilon_{0}}{\varepsilon_{0}} .
$$

Once the electric-field vector $\boldsymbol{E}$ is known in the contrasting domain $\mathrm{D}$, the scattered electric field can be computed through (4). Since we assume that we measure the scattered magnetic field, we use $\boldsymbol{H}^{\text {sct }}=\left(i \omega \mu_{0}\right)^{-1} \nabla \times \boldsymbol{E}^{\text {sct }}$ to arrive at

$$
\begin{aligned}
& H_{3}^{\mathrm{sct}}(\boldsymbol{x}) \\
& =i \omega \varepsilon_{0} \int_{\boldsymbol{x}^{\prime} \in \mathbf{D}} \partial_{2}\left[\frac{i}{4} H_{0}^{(1)}\left(k_{0}\left|\boldsymbol{x}-\boldsymbol{x}^{\prime}\right|\right)\right] \chi\left(\boldsymbol{x}^{\prime}\right) E_{1}\left(\boldsymbol{x}^{\prime}\right) d v \\
& \quad-i \omega \varepsilon_{0} \int_{\boldsymbol{x}^{\prime} \in \mathbf{D}} \partial_{1}\left[\frac{i}{4} H_{0}^{(1)}\left(k_{0}\left|\boldsymbol{x}-\boldsymbol{x}^{\prime}\right|\right)\right] \chi\left(\boldsymbol{x}^{\prime}\right) E_{2}\left(\boldsymbol{x}^{\prime}\right) d v
\end{aligned}
$$

\section{A. Integral Equation for the Magnetic Field}

We note that after substituting

$$
\begin{aligned}
& E_{1}=-(i \omega \varepsilon)^{-1} \partial_{2} H_{3} \\
& E_{2}=(i \omega \varepsilon)^{-1} \partial_{1} H_{3}
\end{aligned}
$$

in the right-hand side of (6), we obtain the integral representation used by Lixin et al. [4], [5], viz.,

$$
H_{3}^{\text {sct }}(\boldsymbol{x})=\int_{\boldsymbol{x}^{\prime} \in \mathbf{D}} \mathcal{M}\left(\boldsymbol{x}^{\prime}\right) \nabla^{\prime}\left[\frac{i}{4} H_{0}^{(1)}\left(k_{0}\left|\boldsymbol{x}-\boldsymbol{x}^{\prime}\right|\right)\right] \nabla^{\prime} H_{3}\left(\boldsymbol{x}^{\prime}\right) d v
$$

in which

$$
\mathcal{M}(\boldsymbol{x})=\frac{\varepsilon(\boldsymbol{x})-\varepsilon_{0}}{\varepsilon(\boldsymbol{x})}
$$

and where $\nabla^{\prime}$ denotes the spatial differentiation with respect to $\boldsymbol{x}^{\prime}$. Using (3) in (8) and taking the point of observation in $\mathrm{D}$, we arrive at

$$
\begin{gathered}
H_{3}(\boldsymbol{x})-\int_{\boldsymbol{x}^{\prime} \in \mathbf{D}} \mathcal{M}\left(\boldsymbol{x}^{\prime}\right) \nabla^{\prime}\left[\frac{i}{4} H_{0}^{(1)}\left(k_{0}\left|\boldsymbol{x}-\boldsymbol{x}^{\prime}\right|\right)\right] \cdot \nabla^{\prime} H_{3}\left(\boldsymbol{x}^{\prime}\right) d v \\
=H_{3}^{\mathrm{inc}}(\boldsymbol{x}), \quad \boldsymbol{x} \in \text { D. }
\end{gathered}
$$

This is an integral equation for $H_{3}$ and $\nabla H_{3}$. Although these quantities are coupled through a spatial differentiation, we prefer an integral equation in which inside and outside the integral the same unknown occurs.

\section{B. Integral Equation for the Electric-Field Vector}

Using (3) in (4) and taking the point of observation in D, we arrive at

$$
\begin{array}{r}
\boldsymbol{E}(\boldsymbol{x})-\left(k_{0}^{2}+\nabla \nabla \cdot\right) \int_{\boldsymbol{x}^{\prime} \in \mathbf{D}} \frac{i}{4} H_{0}^{(1)}\left(k_{0}\left|\boldsymbol{x}-\boldsymbol{x}^{\prime}\right|\right) \chi\left(\boldsymbol{x}^{\prime}\right) \boldsymbol{E}\left(\boldsymbol{x}^{\prime}\right) d v \\
=\boldsymbol{E}^{\text {inc }}(\boldsymbol{x}), \quad \boldsymbol{x} \in \mathrm{D} . \quad
\end{array}
$$

The spatial differentiations in this integral equation can simply be handled by a technique successfully used by Zwamborn and Van den Berg [6]-[10]. In this technique, the gradient operator is handled by testing the integral equation with rooftop functions and, via partial integration, this spatial differentiation is moved to operate on the testing functions. Subsequently, the integral over D is linearly interpolated, after which the divergence operator can be taken. This technique has proven to solve two- and three-dimensional scattering problems fairly accurate, and we shall use this method here as well. We follow a slightly different method and take the integral equation (11) for further consideration. Once we have solved $\boldsymbol{E}$ in D, the scattered magnetic field follows from (6).

Before discussing the discretization procedure of the integral equation, we first write it as

$$
E_{\kappa}-k_{0}^{2} A_{\kappa}-B_{\kappa}=E_{\kappa}^{\mathrm{inc}}, \quad\left(x_{1}, x_{2}\right) \in \mathbf{D}, \quad \kappa \in\{1,2\} .
$$

where the vector

$$
\boldsymbol{B}=\left\{B_{1}\left(x_{1}, x_{2}\right), B_{2}\left(x_{1}, x_{2}\right)\right\}
$$


is given by

$$
B_{\kappa}=\partial_{\kappa}\left[\partial_{1} A_{1}+\partial_{2} A_{2}\right], \quad \kappa \in\{1,2\}
$$

and where the vector potential

$$
\boldsymbol{A}=\left\{A_{1}\left(x_{1}, x_{2}\right), A_{2}\left(x_{1}, x_{2}\right)\right\}
$$

is given by

$$
\begin{aligned}
A\left(x_{1}, x_{2}\right)=\int_{\left(x_{1}^{\prime}, x_{2}^{\prime}\right) \in \mathbf{D}} G\left(x_{1}-x_{1}^{\prime}, x_{2}-x_{2}^{\prime}\right) \\
\cdot \chi\left(x_{1}^{\prime}, x_{2}^{\prime}\right) \boldsymbol{E}\left(x_{1}^{\prime}, x_{2}^{\prime}\right) d x_{1}^{\prime} d x_{2}^{\prime}
\end{aligned}
$$

and the Green function is given by

$$
G\left(x_{1}-x_{1}^{\prime}, x_{2}-x_{2}^{\prime}\right)=\frac{i}{4} H_{0}^{(1)}\left(k_{0}\left|x-x^{\prime}\right|\right) .
$$

\section{DiscretiZATION Procedure}

We assume that the domain $\mathbf{D}$ is a rectangular domain with boundaries along the $x_{1}$ - and $x_{2}$-directions. We discretize the domain in a rectangular mesh. The mesh is uniformly spaced in the $x_{1}$ - and $x_{2}$-directions. The rectangular subdomains with a width of $\Delta x_{1}$ in the $x_{1}$-direction and $\Delta x_{2}$ in the $x_{2}$-direction are given by

$$
\begin{aligned}
\mathbf{D}_{m, n}=\{ & \left(x_{1}, x_{2}\right) \in \mathbf{R}^{2} \mid x_{1 ; m}-\Delta x_{1} / 2<x_{1}<x_{1 ; m} \\
& \left.+\Delta x_{1} / 2, x_{2 ; n}-\Delta x_{2} / 2<x_{2}<x_{2 ; n}+\Delta x_{2} / 2\right\}, \\
& m=1, \cdots, M, \quad n=1, \cdots, N \quad(18)
\end{aligned}
$$

where

$$
\begin{array}{ll}
x_{1 ; m}=x_{1 ; 1 / 2}+\left(m-\frac{1}{2}\right) \Delta x_{1}, & m=1, \cdots, M \\
x_{2 ; n}=x_{2 ; 1 / 2}+\left(n-\frac{1}{2}\right) \Delta x_{2}, & n=1, \cdots, N
\end{array}
$$

in which $x_{1 ; 1 / 2}$ is the lower $x_{1}$ bound of the contrasting domain $\mathrm{D}$, while $x_{2 ; 1 / 2}$ is its lower $x_{2}$ bound. In each subdomain $\mathbf{D}_{m, n}$ with center points $\left(x_{1 ; m}, x_{2 ; n}\right)$, we assume the contrast $\chi$ to be constant with value $\chi_{m, n}$.

We define two sequences of basis functions over the domain $\mathbf{D}$, viz., a sequence $\psi_{m, n}^{(1)}\left(x_{1}, x_{2}\right)$ that is continuous in the $x_{1}$-direction and may jump at discontinuities of the material distribution in the $x_{2}$-direction, and a sequence $\psi_{m, n}^{(2)}\left(x_{1}, x_{2}\right)$ that is continuous in the $x_{2}$-direction and may jump at discontinuities of the material distribution in the $x_{1}$-direction. The most simple basis functions which meet these requirements are the rooftop functions [6]-[10].

To this order, we define the pulse function

$$
\Pi(y \mid \Delta y)= \begin{cases}1, & \text { if }-\frac{1}{2} \Delta y<y<\frac{1}{2} \Delta y \\ \frac{1}{2}, & \text { if }|y|=\frac{1}{\Delta} y \\ 0, & \text { elsewhere }\end{cases}
$$

with support $\Delta y$, and the rooftop function

$$
\Lambda(y \mid \Delta y)= \begin{cases}\left(1+\frac{y}{\Delta y}\right), & \text { if }-\Delta y<y<0 \\ \left(1-\frac{y}{\Delta y}\right), & \text { if } 0 \leq y<\Delta y \\ 0, & \text { elsewhere }\end{cases}
$$

The expansion functions are now defined as

$$
\psi_{m, n}^{(1)}\left(x_{1}, x_{2}\right)=\Lambda\left(x_{1}-x_{1 ; m} \mid \Delta x_{1}\right) \Pi\left(x_{2}-x_{2 ; n} \mid \Delta x_{2}\right)
$$

for $m=1, \cdots, M$ and $n=1, \cdots, N$, and

$$
\psi_{m, n}^{(2)}\left(x_{1}, x_{2}\right)=\Pi\left(x_{1}-x_{1 ; m} \mid \Delta x_{1}\right) \Lambda\left(x_{2}-x_{2 ; n} \mid \Delta x_{2}\right)
$$

for $m=1, \cdots, M$ and $n=1, \cdots, N$. Let us further define the quantities

$$
\begin{aligned}
& E_{\kappa ; m, n}=E_{\kappa}\left(x_{1 ; m}, x_{2 ; n}\right) \\
& B_{\kappa ; m, n}=B_{\kappa}\left(x_{1 ; m}, x_{2 ; n}\right) \\
& A_{\kappa ; m, n}=A_{\kappa}\left(x_{1 ; m}, x_{2 ; n}\right)
\end{aligned}
$$

for $m=1, \cdots, M, n=1, \cdots, N$, and $\kappa \in\{1,2\}$. Then, (12) is discretized as

$$
E_{\kappa ; m, n}-k_{0}^{2} A_{\kappa ; m, n}-B_{\kappa ; m, n} E_{\kappa ; m, n}^{\mathrm{inc}}, \quad \kappa \in\{1,2\} .
$$

Using the expansion functions defined in (22) and (23), (12) is replaced by its weak version

$$
=\frac{\int_{x \in \mathbf{D}}^{B_{\kappa ; m, n}} \psi_{m, n}^{(\kappa)}\left(x_{1}, x_{2}\right) \partial_{\kappa}\left[\partial_{1} A_{1}+\partial_{2} A_{2}\right] d v}{\int_{\boldsymbol{x} \in \mathbf{D}} \psi_{m, n}^{(\kappa)}\left(x_{1}, x_{2}\right) d v}, \quad \kappa \in\{1,2\}
$$

while the vector potential $\boldsymbol{A}$ is expanded as

$$
A_{\kappa}\left(x_{1}, x_{2}\right)=\sum_{m, n} A_{\kappa ; m, n} \psi_{m, n}^{(\kappa)}\left(x_{1}, x_{2}\right), \quad \kappa \in\{1,2\} .
$$

We then arrive at

$$
B_{\kappa ; m, n}=\sum_{\nu=1}^{2} \sum_{p=1}^{M} \sum_{q=1}^{N} a_{m, n, p, q}^{(\kappa, \nu)} A_{\nu ; p, q}, \quad \kappa \in\{1,2\}
$$

where

$$
\begin{aligned}
a_{m, n, p, q}^{(1,1)}= & \left(\Delta x_{1}\right)^{-2}\left(\delta_{p, m+1}-2 \delta_{p, m}+\delta_{p, m-1}\right) \delta_{q, n}, \\
a_{m, n, p, q}^{(1,2)}= & a_{m, n, p, q}^{(2,1)} \\
= & \frac{1}{4}\left(\Delta x_{1} \Delta x_{2}\right)^{-1}\left(\delta_{p, m-1}-\delta_{p, m+1}\right) \\
& \cdot\left(\delta_{q, n-1}-\delta_{q, n+1}\right) \\
a_{m, n, p, q}^{(2,2)}= & \left(\Delta x_{2}\right)^{-2}\left(\delta_{q, n+1}-2 \delta_{q, n}+\delta_{q, n-1}\right) \delta_{p, m} .
\end{aligned}
$$

Hence, the expressions for $B_{\kappa ; m, n}$ become

$$
\begin{aligned}
B_{1 ; m, n}= & \left(\Delta x_{1}\right)^{-2}\left(A_{1 ; m-1, n}-2 A_{1 ; m, n}+A_{1 ; m+1, n}\right) \\
& +\frac{1}{4}\left(\Delta x_{1} \Delta x_{2}\right)^{-1}\left(A_{2 ; m-1, n-1}-A_{2 ; m-1, n+1}\right. \\
- & \left.A_{2 ; m+1, n-1}+A_{2, m+1, n+1}\right) \\
B_{2 ; m, n}= & \left(\Delta x_{2}\right)^{-2}\left(A_{2 ; m, n-1}-2 A_{2 ; m, n}+A_{2 ; m, n+1}\right) \\
& +\frac{1}{4}\left(\Delta x_{1} \Delta x_{2}\right)^{-1}\left(A_{1 ; m-1, n-1}-A_{1 ; m+1, n-1}\right. \\
& \left.-A_{1 ; m-1, n+1}+A_{1, m+1, n+1}\right) .
\end{aligned}
$$


Note that the result in (34) and (35) is identical to the one if we had replaced (14) directly by its finite-difference approximation.

Next, we have to replace the continuous representation of the vector potential $\boldsymbol{A}$ by a discrete one. In order to cope with the singularity of the Green function, we also take the weak form by replacing it by the circular mean of the vector potential. We integrate $A_{\kappa}$ over a circular domain with the center at the point $\left(x_{1 ; m}, x_{2 ; n}\right)$. The radius of these patches is taken to be $\frac{1}{2} \Delta x=\frac{1}{2} \min \left(\Delta x_{1}, \Delta x_{2}\right)$. The results are divided by the surface area $\pi\left(\frac{1}{2} \Delta x\right)^{2}$. We then may write

$$
\begin{aligned}
A_{\kappa ; m, n}= & A_{\kappa}\left(x_{1 ; m}, x_{2 ; n}\right) \\
= & \int_{\left(x_{1}^{\prime}, x_{2}^{\prime}\right) \in \mathbf{D}} \mathcal{G}\left(x_{1 ; m}-x_{1}^{\prime}, x_{2 ; n}-x_{2}^{\prime}\right) \chi\left(x_{1}^{\prime}, x_{2}^{\prime}\right) \\
& \cdot E_{\kappa}\left(x_{1}^{\prime}, x_{2}^{\prime}\right) d x_{1}^{\prime} d x_{2}^{\prime}, \quad \text { with } \kappa \in\{1,2\} \quad
\end{aligned}
$$

where we have interchanged the order of integrations, such that

$$
\begin{aligned}
\mathcal{G}\left(x_{1}, x_{2}\right)= & \frac{4}{\pi(\Delta x)^{2}} \\
& \cdot \int_{\left(x_{1}^{\prime 2}+x_{2}^{\prime 2}\right)^{1 / 2}<(1 / 2) \Delta x} G\left(x_{1}+x_{1}^{\prime}, x_{2}+x_{2}^{\prime}\right) \\
& \cdot d x_{1}^{\prime} d x_{2}^{\prime} \\
= & \left\{\begin{array}{c}
\frac{i}{k_{0} \Delta x} J_{1}\left(\frac{1}{2} k_{0} \Delta x\right) H_{0}^{(1)}\left[k_{0}\left(x_{1}^{2}+x_{2}^{2}\right)^{1 / 2}\right], \\
\frac{i}{k_{0} \Delta x}\left[\begin{array}{c}
\text { when }\left(x_{1}^{2}+x_{2}^{2}\right)^{1 / 2}>\frac{1}{2} \Delta x \\
\text { when } x_{1}=x_{2}=0 .
\end{array}\right.
\end{array}\right.
\end{aligned}
$$

In fact, $\mathcal{G}$ is the mean value of the Green function over a circular domain with the center at $\left(x_{1}, x_{2}\right)$. After this weakening procedure, we are now able to compute the integrals of (36) numerically. In view of the functional properties of $E_{\kappa}$, we approximate the integral of (36) using a midpoint rule in the $x_{1}$ - and $x_{2}$-directions. We then arrive at

$$
\begin{array}{r}
A_{\kappa ; m, n}=\Delta x_{1} \Delta x_{2} \sum_{m^{\prime}=1}^{M} \sum_{n^{\prime}=2}^{N} \mathcal{G}\left(x_{1 ; m}-x_{1 ; m^{\prime}}, x_{2 ; n}-x_{2 ; n^{\prime}}\right) \\
\cdot \chi_{m^{\prime}, n^{\prime}} E_{\kappa ; m^{\prime}, n^{\prime}}
\end{array}
$$

with $\kappa \in\{1,2\}$ and for $m=1, \cdots, M$ and $n=1, \cdots, N$. Note that $A_{\kappa ; m, n}$ are discrete convolutions and can efficiently be computed by fast Fourier transform (FFT) routines.

\section{FORWARD SCATTERING PROBLEM}

When we substitute (38) in (30) and use the result in (27), we obtain a linear system of equations for $E_{\kappa ; m, n}$, when the material contrast $\chi$ is known. This system of equations can be solved by a conjugate gradient scheme. In such a scheme, we need the representation of the operator, definition of the norm and inner product, and representation of the adjoint operator. The operator expression $\boldsymbol{L} \boldsymbol{E}$ is directly obtained from (27),
(30), and (38) as

$$
\begin{aligned}
& (\boldsymbol{L E})_{\kappa ; p, q} \\
& \quad=E_{\kappa ; p, q}-k_{0}^{2} A_{\kappa ; p, q}-\sum_{\nu=1}^{2} \sum_{m=1}^{M} \sum_{n=1}^{N} a_{p, q, m, n}^{(\kappa, \nu)} A_{\nu ; m, n}
\end{aligned}
$$

for $p=1, \cdots, M$ and $q=1, \cdots, N$, and where $A_{\kappa ; m, n}$ directly follows from (38).

The norm on $\mathbf{D}$ is defined as

$$
\|\boldsymbol{E}\|_{\mathbf{D}}^{2}=\langle\boldsymbol{E}, \boldsymbol{E}\rangle_{\mathbf{D}}=\sum_{\nu=1}^{2} \sum_{p=1}^{M} \sum_{q=1}^{N} E_{\nu ; p, q} \bar{E}_{\nu ; p, q}
$$

where the overbar denotes the complex conjugate. The adjoint operator is defined through the relation

$$
\langle\boldsymbol{r}, L \boldsymbol{E}\rangle_{\mathbf{D}}=\left\langle\boldsymbol{L}^{\star} \boldsymbol{r}, \boldsymbol{E}\right\rangle_{\mathbf{D}}
$$

Substituting the expression of the operator $\boldsymbol{L} \boldsymbol{E}$ in the lefthand side of (41) and interchanging the various summations, the adjoint operator is recognized as

$$
\left(\boldsymbol{L}^{\star} \boldsymbol{r}\right)_{\kappa ; p, q}=r_{\kappa ; p, q}-\bar{\chi}_{p, q} C_{\kappa ; p, q}
$$

for $p=1, \cdots, M$ and $q=1, \cdots, N$, in which

$$
\begin{gathered}
C_{\kappa ; p, q}=\Delta x_{1} \Delta x_{2} \sum_{p^{\prime}=0}^{M+1} \sum_{q^{\prime}=0}^{N+1} \overline{\mathcal{G}}\left(x_{1 ; p}-x_{1 ; p^{\prime}}, x_{2 ; q}-x_{2 ; q^{\prime}}\right) \\
\cdot F_{\kappa ; p^{\prime}, q^{\prime}}
\end{gathered}
$$

where

$$
F_{\kappa ; p, q}=k_{0}^{2} r_{\kappa ; p, q}+\sum_{\nu=1}^{2} \sum_{m=1}^{M} \sum_{n=1}^{N} a_{m, n, p, q}^{(\kappa, \nu)} r_{\nu ; m, n} .
$$

The latter expressions may be written explicitly as

$$
\begin{gathered}
F_{1 ; p, q}=k_{0}^{2} r_{1 ; p, q}+\left(\Delta x_{1}\right)^{-2}\left(r_{1 ; p-1, q}-2 r_{1 ; p, q}+r_{1 ; p+1, q}\right) \\
+\frac{1}{4}\left(\Delta x_{1} \Delta x_{2}\right)^{-1}\left(r_{2 ; p-1, q-1}-r_{2 ; p-1, q+1}\right. \\
\left.-r_{2 ; p+1, q-1}+r_{2, p+1, q+1}\right)
\end{gathered}
$$

$$
\begin{gathered}
F_{2 ; p, q}=k_{0}^{2} r_{2 ; p, q}+\left(\Delta x_{2}\right)^{-2}\left(r_{2 ; p, q-1}-2 r_{2 ; p, q}+r_{2 ; p, q+1}\right) \\
+\frac{1}{4}\left(\Delta x_{1} \Delta x_{2}\right)^{-1}\left(r_{1 ; p-1, q-1}-r_{1 ; p+1, q-1}\right. \\
\left.-r_{1 ; p-1, q+1}+r_{1, p+1, q+1}\right) .
\end{gathered}
$$

Since according to (43) $p^{\prime}$ runs from 0 to $M+1$ and $q^{\prime}$ runs from 0 to $N+1$, we set in (45) and (46)

$$
\begin{array}{lll}
r_{\kappa ; p, q}=0, & \text { for } p=-1,0, M+1, M+2 & \forall q \\
r_{\kappa ; p, q}=0, & \text { for } q=-1,0, N+1, N+2 & \forall p .
\end{array}
$$

Note that $C_{\kappa ; p, q}$ is a discrete convolution in $p$ and $q$, and these convolutions can be computed efficiently by FFT routines [11].

With these definitions, we are now able to apply a conjugate gradient iterative scheme to solve the equations

$$
(\boldsymbol{L E})_{\kappa ; p, q}=E_{\kappa ; p, q}^{\mathrm{inc}}, \quad p=1, \cdots, M, \quad q=1, \cdots, N .
$$


Once the normalized error

$$
\mathrm{ERR}=\frac{\left\|\boldsymbol{L} \boldsymbol{E}-\boldsymbol{E}^{\mathrm{inc}}\right\|_{\mathrm{D}}}{\left\|\boldsymbol{E}^{\mathrm{inc}}\right\|_{\mathrm{D}}}
$$

is small enough, the approximate solution of $\boldsymbol{E}$ is substituted in (6) to arrive at the scattered magnetic field at the receiver points $\boldsymbol{x}^{(r)}$, with $r=1,2, \cdots, R$,

$$
H_{3}^{\text {sct }}\left(\boldsymbol{x}^{(r)}\right)=i \omega\left(\partial_{2}^{(r)} A_{1}-\partial_{1}^{(r)} A_{2}\right)
$$

in which $\partial_{1}^{(r)}$ and $\partial_{2}^{(r)}$ denote the spatial differentiations in regard to $x_{1}^{(r)}$ and $x_{2}^{(r)}$, respectively.

\section{INVERSE SCATTERING PROBLEM}

We now assume that the inhomogeneous object $\mathbf{D}$ is irradiated successively by a number $(s=1,2, \cdots, S)$ of known incident fields. For each excitation, the direct scattering problem may be reformulated as the domain integral equation [cf. (48)]

$$
\boldsymbol{L} \boldsymbol{E}^{(s)}=\boldsymbol{E}^{\text {inc, }(s)}, \quad \text { on D }
$$

where the operator $\boldsymbol{L}$ depends on the material contrast $\chi$. To show this explicitly, we write this equation as

$$
\boldsymbol{E}^{(s)}-V \chi \boldsymbol{E}^{(s)}=\boldsymbol{E}^{\text {inc, },(s)}, \quad \text { on D }
$$

where

$$
\left(\boldsymbol{V} \chi \boldsymbol{E}^{(s)}\right)_{\kappa ; p, q}=k_{0}^{2} A_{\kappa ; p, q}^{(s)}+\sum_{\nu=1}^{2} \sum_{m=1}^{M} \sum_{n=1}^{N} a_{p, q, m, n}^{(\kappa, \nu)} A_{\nu ; m, n}^{(s)}
$$

for $p=1, \cdots, M$ and $q=1, \cdots, N$. In (53), $A_{\nu ; m, n}^{(s)}$ follows from (38) by replacing $E_{\kappa ; m, n}$ by $E_{\kappa ; m, n}^{(s)}$. Equation (52) is denoted as the OBJECT EQUATION that holds in the object domain D.

Further, in the inverse problem, $H_{3}$ is known at the measurement points $\boldsymbol{x}^{(r)}$. We assume that all the measurement points are located in the data domain $S$. Hence, we write (50) also in the shorthand notation

$$
W \chi \cdot \boldsymbol{E}^{(s)}=H_{3}^{(s)}\left(\boldsymbol{x}^{(r)}\right), \quad \text { on } \mathcal{S}
$$

where $W \chi \cdot \boldsymbol{E}^{(s)}$ is equal to the right-hand side of (50) after replacing $\boldsymbol{E}$ by $\boldsymbol{E}^{(s)}$ and $H_{3}^{\text {sct }}$ by $H_{3}^{(s)}$. Equation (54) is denoted as the DATA EQUATION that holds in the data domain $\mathcal{S}$.

\section{A. Reconstruction of the Complex Contrast $\chi$}

The inverse problem is that of finding the material contrast $\chi$ from (52) and (54). Since the electric-field vector $\boldsymbol{E}^{(s)}$ is also unknown, we propose the iterative construction of sequences $\left\{\boldsymbol{E}_{k}^{(s)}\right\}$ and $\left\{\chi_{k}\right\}$ as follows:

$$
\begin{aligned}
\boldsymbol{E}_{k}^{(s)} & =\boldsymbol{E}_{k-1}^{(s)}+\alpha_{k} \boldsymbol{e}_{k}^{(s)} \\
\chi_{k} & =\chi_{k-1}+\beta_{k} m_{k}, \quad k=1,2, \cdots .
\end{aligned}
$$

For each $k$, the functions $\boldsymbol{e}_{k}^{(s)}$ and $m_{k}$ are update directions for the electric-field vector $\boldsymbol{E}^{(s)}$ and the material contrast $\chi$, respectively, while the complex parameters $\alpha_{k}$ and $\beta_{k}$ are weights to be determined. The residual errors at each step in the object equation and data equation are defined as

$$
\begin{aligned}
\boldsymbol{r}_{k}^{(s)} & =\boldsymbol{E}^{\mathrm{inc},(s)}-\boldsymbol{E}_{k}^{(s)}+V \chi_{k} \boldsymbol{E}_{k}^{(s)} \\
\rho_{k}^{(s)} & =H_{3}^{(s)}-\mathbf{W} \chi_{k} \cdot \boldsymbol{E}_{k}^{(s)} .
\end{aligned}
$$

The constants $\alpha_{k}$ and $\beta_{k}$ are determined by minimizing the value of the cost functional

$$
F_{k}=w_{\mathbf{D}} \sum_{s=1}^{S}\left\|\boldsymbol{r}_{k}^{(s)}\right\|_{\mathbf{D}}^{2}+w_{\mathcal{S}} \sum_{s=1}^{S}\left\|\rho_{k}^{(s)}\right\|_{\mathcal{S}}^{2}
$$

in which

$$
\begin{aligned}
& w_{\mathbf{D}}=\left(\sum_{s=1}^{S}\left\|\boldsymbol{E}^{\text {inc, }(s)}\right\|_{\mathbf{D}}^{2}\right)^{-1} \\
& w_{\mathcal{S}}=\left(\sum_{s=1}^{S}\left\|H_{3}^{(s)}\right\|_{\mathcal{S}}^{2}\right)^{-1} .
\end{aligned}
$$

Here, the norm $\|\cdot\|_{\mathbf{D}}$ in the object domain is defined in (40), while the norm in the data domain is defined as

$$
\left\|H_{3}\right\|_{\mathcal{S}}^{2}=\sum_{\boldsymbol{x}^{r} \in \mathcal{S}}\left|H_{3}\left(\boldsymbol{x}^{r}\right)\right|^{2} .
$$

Substitution of (55)-(57) in the cost functional of (58) results in an expression involving terms determined at the $(k-1)$ st step, the directions $\boldsymbol{e}_{k}^{(s)}$ and $m_{k}$, and the two parameters $\alpha_{k}$ and $\beta_{k}$. Once the directions $\boldsymbol{e}_{k}^{(s)}$ and $m_{k}$ are chosen, we have a nonlinear expression in $\alpha_{k}$ and $\beta_{k}$. The values of the parameters $\alpha_{k}$ and $\beta_{k}$ are determined by requiring $F_{k}$ to be a minimum. Minimization of the quantity $F_{k}$ is accomplished by solving this nonlinear problem in $\alpha_{k}$ and $\beta_{k}$ using a standard conjugate gradient method.

The update directions $\boldsymbol{e}_{k}^{(s)}$ and $m_{k}$ are chosen as the Polak-Ribière conjugate gradient directions, namely,

and

$$
\boldsymbol{e}_{k}^{(s)}=\partial \boldsymbol{e}_{k}^{(s)}+\gamma_{k}^{e} \boldsymbol{e}_{k-1}^{(s)}
$$

$$
m_{k}=\partial m_{k}+\gamma_{k}^{m} m_{k-1}
$$

where

$$
\begin{gathered}
\gamma_{k}^{\boldsymbol{e}}=\frac{\sum_{s=1}^{S}\left\langle\partial \boldsymbol{e}_{k}^{(s)}, \partial \boldsymbol{e}_{k}^{(s)}-\partial \boldsymbol{e}_{k-1}^{(s)}\right\rangle_{\mathbf{D}}}{\sum_{s=1}^{S}\left\|\partial \boldsymbol{e}_{k-1}^{(s)}\right\|_{\mathbf{D}}^{2}} \\
\gamma_{k}^{m}=\frac{\left\langle\partial m_{k}, \partial m_{k}-\partial m_{k-1}\right\rangle_{\mathbf{D}}}{\left\|\partial m_{k-1}\right\|_{\mathbf{D}}^{2}}
\end{gathered}
$$

and the gradients are given by

$$
\partial \boldsymbol{e}_{k}^{(s)}=w_{\mathbf{D}}\left(\boldsymbol{r}_{k-1}^{(s)}-\bar{\chi}_{k-1} \boldsymbol{V}^{\star} \boldsymbol{r}_{k-1}^{(s)}\right)+w_{\mathcal{S}} \bar{\chi}_{k-1} \boldsymbol{W}^{\star} \rho_{k-1}^{(s)}
$$

and

$$
\partial m_{k}=-w_{\mathbf{D}} \sum_{s=1}^{S} \overline{\boldsymbol{E}}_{k-1}^{(s)} \cdot \boldsymbol{V}^{\star} \boldsymbol{r}_{k-1}^{(s)}+w_{\mathcal{S}} \sum_{s=1}^{S} \overline{\boldsymbol{E}}_{k-1}^{(s)} W^{\star} \rho_{k-1}^{(s)} .
$$


The various operators are calculated as (omitting the iteration index $k-1$ )

$$
\begin{aligned}
\left(\bar{\chi} \boldsymbol{V}^{\star} \boldsymbol{r}^{(s)}\right)_{\kappa ; p, q} & =\bar{\chi}_{p, q} r_{\kappa ; p, q}^{(s)}+\bar{\chi}_{p, q} C_{\kappa ; p, q}^{(s)} \\
\left(\overline{\boldsymbol{E}}^{(s)} \cdot \boldsymbol{V}^{\star} \boldsymbol{r}^{(s)}\right)_{p, q} & =\sum_{\nu=1}^{2} \bar{E}_{\nu ; p, q}^{(s)} r_{\nu ; p, q}^{(s)}+\bar{E}_{\nu ; p, q}^{(s)} C_{\nu ; p, q}^{(s)} \\
\left(\bar{\chi} \mathbf{W}^{\star} \rho^{(s)}\right)_{\kappa ; p, q} & =\bar{\chi}_{p, q}\left(\boldsymbol{W}^{\star} \rho^{(s)}\right)_{\kappa ; p, q} \\
\left(\overline{\boldsymbol{E}^{(s)}} \cdot \mathbf{W}^{\star} \rho^{(s)}\right)_{p, q} & =\sum_{\nu=1}^{2} \overline{\boldsymbol{E}^{(s)}}{ }_{\nu ; p, q}\left(W^{\star} \rho^{(s)}\right)_{\nu ; p, q}
\end{aligned}
$$

for $p=1, \cdots, M$ and $q=1, \cdots, N$.

\section{B. Reconstruction of a Positive Conductivity Contrast}

In order to perform the reconstruction of a positive conductivity contrast, we can incorporate a priori information about its positivity into our inversion scheme. By introducing a new function $\zeta_{k}$ such that $\chi_{k}=i \zeta_{k}^{2}$ and by updating $\zeta_{k}$ as

$$
\zeta_{k}=\zeta_{k-1}+\beta_{k} \xi_{k}, \quad k=1,2, \cdots
$$

instead of the contrast $\chi_{k}$, we are able to enforce the positivity of our contrast function $\chi$. The residual errors at each step in the object equation and data equation are defined in (57) with $\chi_{k}=i \zeta_{k}^{2}$. The constants $\alpha_{k}$ and $\beta_{k}$ are determined again by minimizing the cost functional $F_{k}$ defined in (58).

The update directions $\boldsymbol{e}_{k}^{(s)}$ and $\xi_{k}$ are chosen as the Polak-Ribière conjugate gradient directions given by the first equation in (62) and by

and

$$
\xi_{k}=\partial \xi_{k}+\gamma_{k}^{\xi} \xi_{k-1}
$$

$$
\gamma_{k}^{\xi}=\frac{\left\langle\partial \xi_{k}, \partial \xi_{k}-\partial \xi_{k-1}\right\rangle_{\mathbf{D}}}{\left\|\partial \xi_{k-1}\right\|_{\mathbf{D}}^{2}}
$$

where $\partial \xi_{k}$ is the gradient of the cost function $F_{k}$ with respect to changes in $\zeta$ evaluated at step $(k-1)$, assuming the fields do not change

$$
\partial \zeta_{k}=\partial_{\zeta} F_{k-1}=2 \zeta_{k-1} \operatorname{Im}\left[\partial \chi_{k}\right]
$$

The initial choice in our iterative scheme is determined from an estimate of the contrast sources according to method described in (22)-(28) of Kleinman and Van den Berg [3], which is adapted to our vectorial object and data integral equations.

\section{NUMERICAL EXAMPLES}

In our numerical examples, it was assumed that the unknown scatterer was located entirely within a test square of known dimension, although knowledge of the precise location within the test square was not assumed. All computations were carried out on a $200-\mathrm{MHz}$ Pentium Pro Workstation running Windows NT 4.0. The Fortran code was compiled using a Digital Visual Fortran 5.0 compiler and the computation time for one iteration in the inversion in the most complicated examples was $2.2 \mathrm{~min}$.

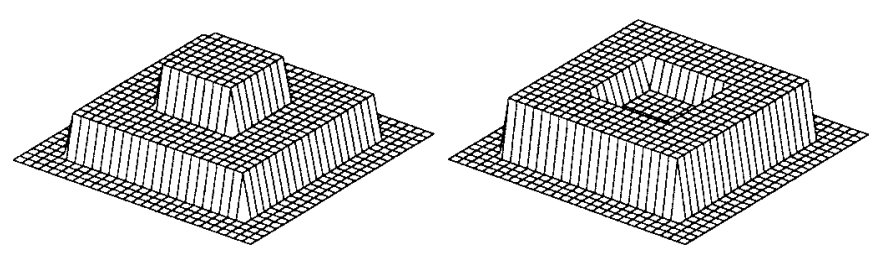

(a)

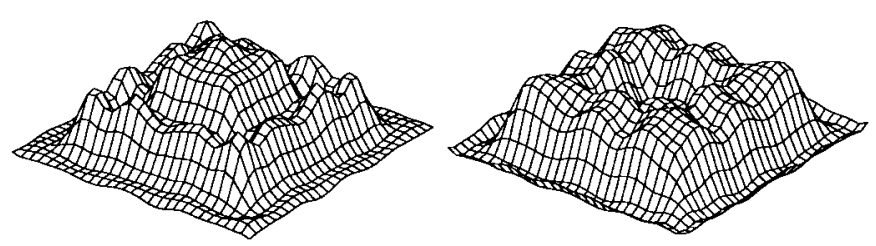

(b)

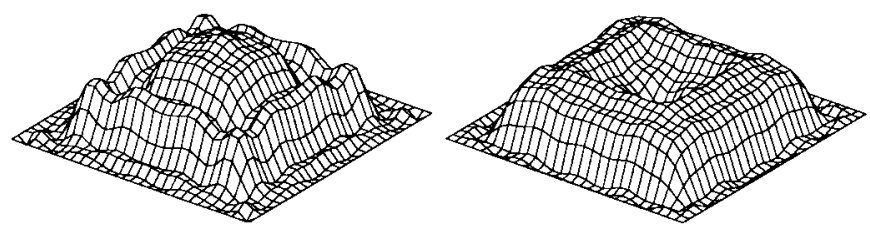

(c)

Fig. 1. (a) The original profile. (b) The reconstructed profile using the magnetic-field integral equation and synthetic data generated by the magnetic-field integral equation. (c) The reconstructed profile using the electric-field integral equation and synthetic data generated by the electric-field integral equation. 128 iterations, 29 stations.

\section{A. Example 1}

We illustrate the TE inversion of a complex contrast with a numerical example, in which the measurement surface $\mathcal{S}$ is chosen to be a circle of radius $9 \lambda$ containing the test domain $\mathrm{D}$, which is a square $(3 \lambda \times 3 \lambda)$ where $\lambda$ is the wavelength in the background medium. The test domain is discretized into $29 \times$ 29 subsquares. The configuration to be reconstructed consists of two concentric square cylinders, an inner cylinder, $\lambda$ by $\lambda$, with complex permittivity $\varepsilon=(1.6+0.2 i) \varepsilon_{0}$ surrounded by an outer cylinder $2 \lambda \times 2 \lambda$, with complex permittivity $\varepsilon=(1.3+$ $0.4 i) \varepsilon_{0}$, as shown in Fig. 1(a). In our first example, we assume that 29 stations $(J=29)$ are located uniformly on the surface $S$ with each station serving successively as a line source and all stations acting as receivers. The reconstruction based on the magnetic-field equation [4], [5] is shown in Fig. 1(b), while the reconstruction based on our present approach using the integral equation for the electric-field strength is presented in Fig. 1(c). In both cases, synthetic "measured" data are generated by solving the forward problem using the same type of integral equation as the one used in the inversion scheme.

However, in the reconstruction procedures of Fig. 1, we have the possibility of inadvertently committing the "inverse crime" by using the same numerical method in the inversion algorithm as is used for solving the forward problem to produce the synthetic "measured" data. Therefore, in Figs. 2 and 3, we use the synthetic data generated by the magneticfield integral equation in the inversion procedure based on the electric-field integral equation, while we use the synthetic data generated by the electric-field integral equation in the inversion procedure based on the magnetic-field integral-equation. The 


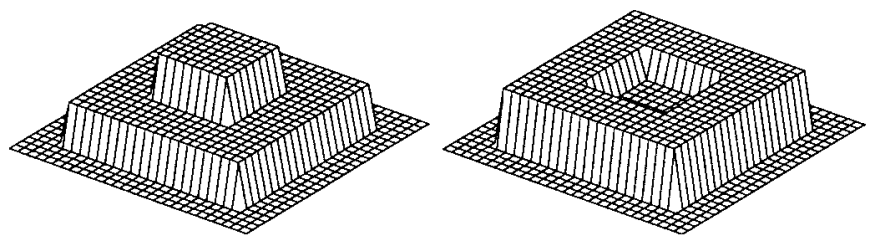

(a)
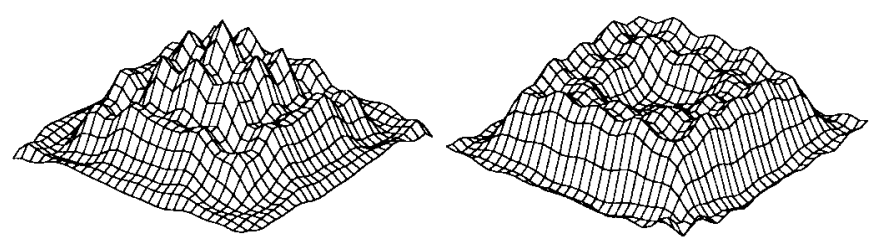

(b)
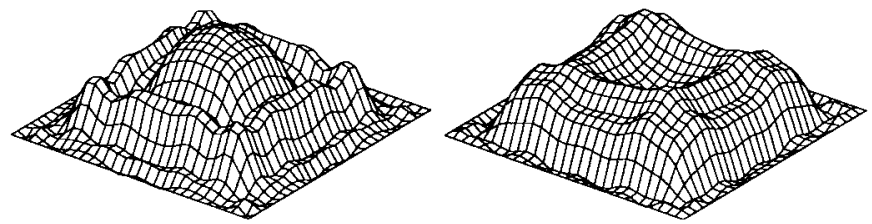

(c)

Fig. 2. (a) The original profile. (b) The reconstructed profile using the magnetic-field integral equation and synthetic data generated by the electric-field integral equation. (c) The reconstructed profile using the electric-field integral equation and synthetic data generated by the magnetic-field integral equation. 128 iterations, 29 stations.
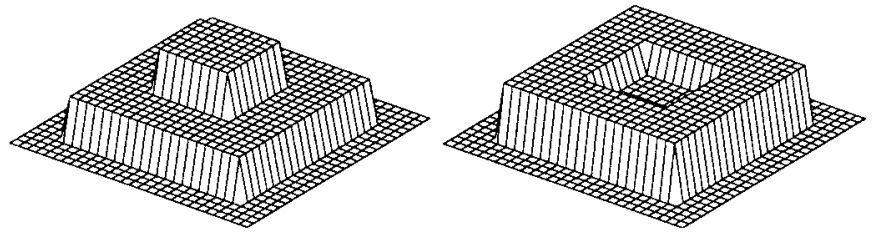

(a)
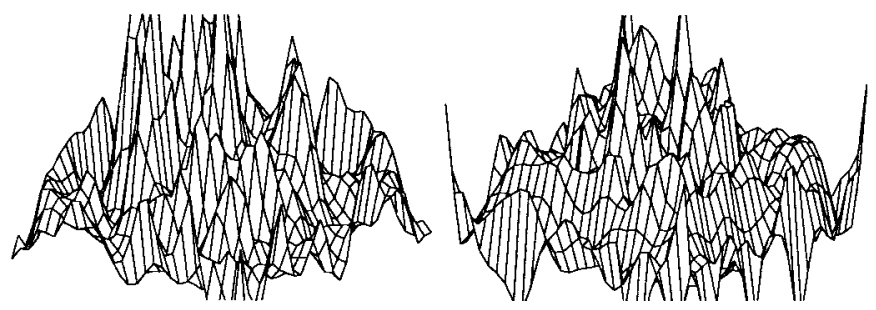

(b)
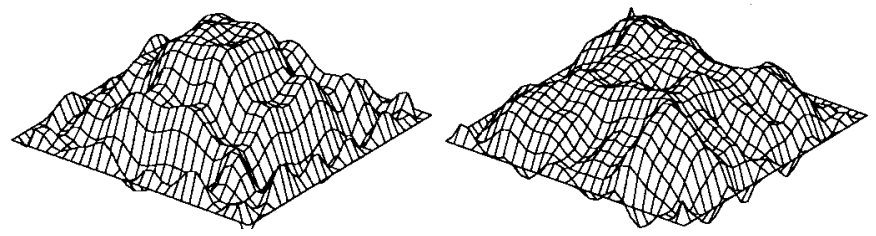

(c)

Fig. 3. Same as Fig. 2, but now only 15 stations.

results for 29 stations are presented in Fig. 2, while the results for only 15 stations are given in Fig. 3. From Fig. 3, we observe that for a limited amount of data, the inversion procedure based on the electric-field integral equation, as presented in this paper, still yields acceptable reconstructions.

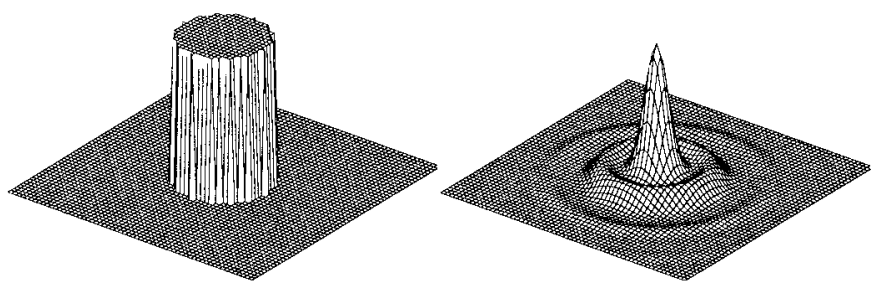

(a)

(b)

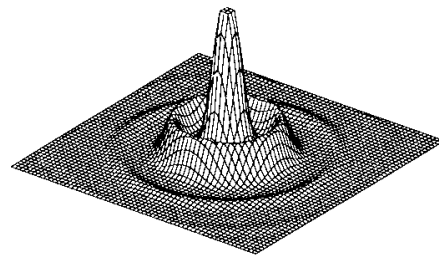

(c)

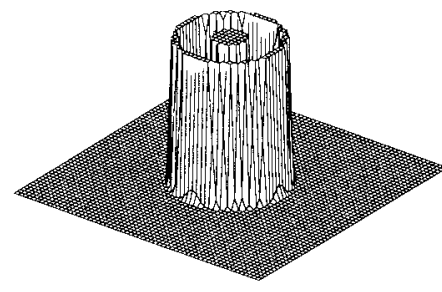

(e)

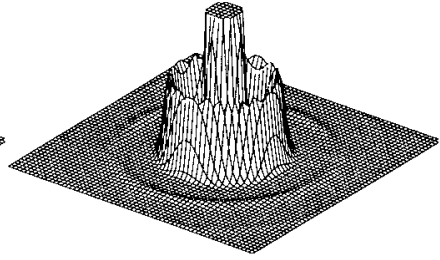

(d)

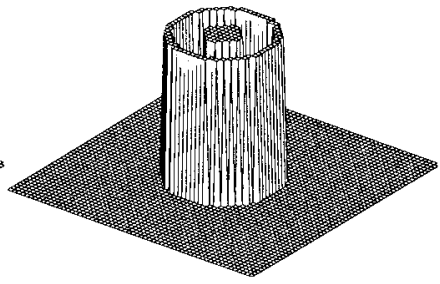

(f)
Fig. 4. (a) The reconstruction of a perfectly conducting circular cylinder with a bounded contrast symmetrically located with respect to the sources and receivers. (b)-(f) A bounded reconstruction after 2, 4, 8, 16, and 32 iterations, respectively.

\section{B. Example $2 a$}

We illustrate the TE inversion of a positive conductivity contrast with a numerical example. In this example, we assume a test square of $61 \times 61$ subsquares of $0.0015 \times 0.0015 \mathrm{~m}^{2}$. In the center of this test square, a perfectly conducting circular cylinder with a radius of $0.0159 \mathrm{~m}$ is located [see Fig. 4(a)]. The unit circle with center point in the center of the test domain is taken as the measurement surface $\mathcal{S}$. On the surface of $\mathcal{S}$, we illuminate the object with 36 uniformly distributed planewave sources and measure the wave field at 18 uniformly distributed receivers. The operating frequency of the sources is $10 \mathrm{GHz}$. The measured data were simulated by solving the direct scattering problem of a perfectly conducting circular cylinder. The analytic solution in terms of Bessel functions has been employed. In this example, the positivity of the conductivity has been enforced in our iterative inversion scheme. As for a perfectly conductive object, the contrast in the reconstruction reaches at some point a value such that the penetration depth of the wavefield is of the order of the mesh width in the testing domain, we can improve the visualization of the boundary of the object by imposing an upper bound to the reconstructed contrast in such a way that the penetration depth of the wavefield is not less than three times the mesh width. When in the iterative procedure the contrast reaches this upper bound, then this value is kept fixed and the gradient of the contrast in this direction is set to zero. For this reason, the reconstruction of the cylinder does not improve after 32 


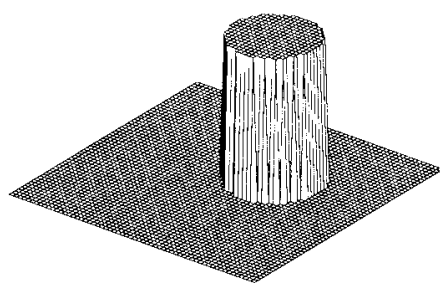

(a)

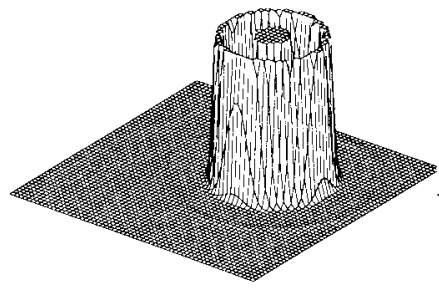

(b)

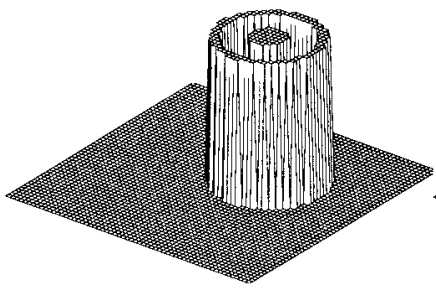

(d)

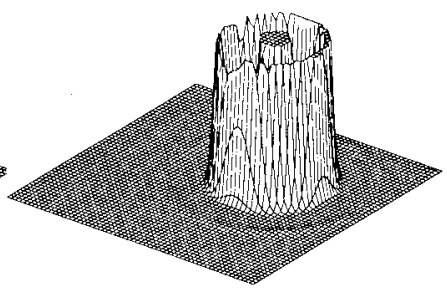

(c)

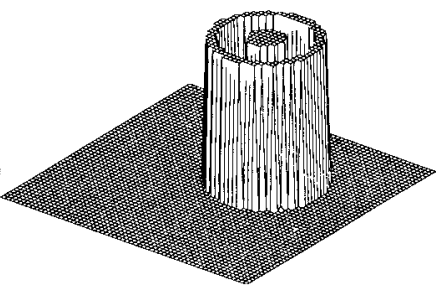

(e)
Fig. 5. The reconstruction of a perfectly conducting circular cylinder with a bounded contrast asymmetrically located with respect to the sources and receivers and depicted in (a). (b) and (d) represent a bounded reconstruction after 16 and 32 iterations, respectively. (c) and (e) represent a bounded reconstruction of the data with $10 \%$ noise added after 16 and 32 iterations, respectively.

iterations because most of the contrast values at the location of the cylinder are set to the upper bound of one in this case. We observe that after only four iterations, the boundary of the object is already clearly visible. Specifically, we observe that after eight iterations, the inner center part of the cylinder already reaches the bounded value of one. At sixteen iterations, we observe that the boundary of the cylinder already has reached its bounded value of one and that incrementation of the number of iterations only gives a little improvement.

\section{Example $2 b$}

In this last example, we assume a test square of $61 \times$ 61 subsquares of $0.0015 \times 0.0015 \mathrm{~m}^{2}$. Out of the center of this test square, a perfectly conducting circular cylinder with a radius of $0.0159 \mathrm{~m}$ is located [see Fig. 5(a)]. The unit circle with the center point in the center of the test domain is taken as the measurement surface $\mathcal{S}$. On the surface of $\mathcal{S}$, we illuminate the object with 36 uniformly distributed plane-wave sources and measure the field at 18 uniformly distributed receivers. The operating frequency of the sources is $10 \mathrm{GHz}$. The measured data were simulated by solving the direct scattering problem of an impenetrable circular cylinder. The analytic solution in terms of Bessel functions has been employed. In this example, the positivity of the conductivity has been enforced in our iterative inversion scheme. The contrast of the cylinder has been bounded again to one. We observe, in the subsequent iterations, the same phenomena as in the previous example. However, we observe that the object in this case is not reconstructed in a symmetric way because the location of the object, with respect to the sources and receivers, is not symmetric in this case. This example shows that not only the reconstruction of the boundary is very well, but that the location of the object in the testing domain is determined precisely. In this example, we also investigated the influence of noise. The results in Fig. 5(c) and (e) show that the method performs well when $10 \%$ noise is added to the data.

\section{ConClusions}

We have developed a nonlinear inversion method for the two-dimensional problem of the scattering of an electromagnetic wave with the magnetic-field vector parallel to the cylindrical structure. The electric-field vector has two components in the transversal plane (TE case). We have shown that a formulation of the inversion procedure in terms of the electric field yields better reconstruction results than an inversion procedure formulated in terms of the magnetic-field vector. The theoretical results in this paper are written in such a form that the necessary changes of the original scalar computer code of the TM case, developed by Kleinman and Van den Berg [3], [5], are limited to a minimum. The results obtained with the TE case look quite the same compared to the results obtained with the TM case [3]. The reason for this is probably that in the TE case, as well as in the TM case, we are measuring a scalar field instead of a vectorial field.

The present solution of the TE case is an intermediate step to the full three-dimensional problem where all the three components of the electric field play their role. In the forward problem, Zwamborn and Van den Berg [8] have shown that the step from the two-dimensional TE case to the full three-dimensional case is rather trivial. All fundamental difficulties of the three-dimensional case are the same in the TE case. Only, in the three-dimensional case, there is extra book keeping in writing the computer code and, obviously for realistic configurations, the computation time increases substantially [9]. It is expected that the same will be true for the inverse problem. Hence, it is a prerequisite to first solve the inverse problem in the TE case. For the latter case, we have described the method based on the modified-gradient method; this avoids the need of a full solution of the forward problem in each iteration of the inverse problem. With this feature, it seems that a three-dimensional inverse problem can be handled with present-day computer power.

\section{REFERENCES}

[1] R. E. Kleinman and P. M. van den Berg, "A modified gradient method for two-dimensional problems in tomography," J. Comput. Appl. Math., vol. 42, pp. 17-35, 1992.

[2] inversion," Radio Sci., vol. 28, pp. 877-884, 1993.

[3] _ "Two-dimensional location and shape reconstruction," Radio Sci., vol. 29, pp. 1157-1169, 1994. 
[4] W. Lixin, R. E. Kleinman, and P. M. van den Berg, "Two-dimensional profile inversion-The TE case," in Proc. Int. Symp. Electromagn. Theory, St. Petersburg, Russia, May 23-26, 1995, pp. 161-162.

[5] _ "Modified gradient profile inversion," in IEEE Antennas Propagat. Soc. Int. Symp., Newport Beach, CA, June 18-23, 1995, pp. $1598-1601$.

[6] A. P. M. Zwamborn and P. M. van den Berg, "A weak form of the conjugate gradient FFT method for two-dimensional TE scattering problems," IEEE Trans. Microwave Theory Tech., vol. 39, pp. 953-960, June 1991.

[7] A. P. M. Zwamborn, "Scattering by objects with electric contrast," Ph.D. dissertation, Dept. Elect. Eng., Delft Univ., Delft, The Netherlands, 1991.

[8] A. P. M. Zwamborn and P. M. van den Berg, "The three-dimensional weak form of the conjugate gradient FFT method for solving scattering problems," IEEE Trans. Microwave Theory Tech., vol. 40, pp. 1757-1766, Mar. 1992.

[9] A. P. M. Zwamborn, P. M. van den Berg, J. Mooibroek, and F. T. C. Koenis, "Computation of three-dimensional electromagnetic field distributions in a human body using the weak form of the CGFFT method," Appl. Comput. Electromagn. Soc. J., Special Issue on Biolelectromagnetic Computations, vol. 7, pp. 26-42, 1992.

[10] A. P. M. Zwamborn and P. M. van den Berg, "Computation of electromagnetic fields inside strongly objects by the weak-conjugategradient fast-Fourier-transform method," J. Opt. Soc., vol. 11, pp. 1414-1421, 1994.

[11] D. Lesselier and B. Duchene, "Wavefield inversion of objects in stratified environments from backpropagation schemes to full solution," in Progress in Electromagnetics Research. London, U.K.: Oxford University Press, 1996, pp. 235-268.

[12] W. H. Press, B. P. Flannery, S. A. Teukolsky, and W. T. Vetterling, Numerical Recipes: The Art of Scientific Computing. Cambridge, U.K.: Cambridge Univ. Press, 1986.

[13] J. H. Richmond, "Scattering by a dielectric cylinder of arbitrary cross section shape," IEEE Trans. Antennas Propagat., vol. AP-13, pp. 334-341, Mar. 1965.

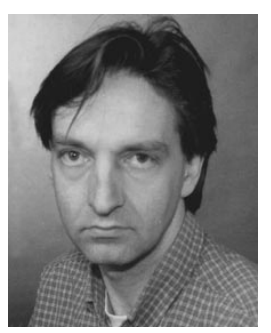

Bert Jan Kooij was born in Amersfoort, The Netherlands, on February 11, 1959. He received the B.Sc. and M.Sc. degrees in electrical engineering, and the Ph.D. degree in technical sciences from Delft University of Technology, Delft, The Netherlands, in 1984, 1986, and 1994, respectively.

Since 1987, he has been a member of the Scientific Staff of the Electromagnetic Research Group, Delft University of Technology, where he has carried out research and taught classes in the area of electromagnetic, as well as acoustic, wave propagation and scattering problems. During a three-month period in 1996, he was a Visiting Scientist at Ecole Supérieure d'Electricité (Supelec), Gif sur Yvette, France. He has worked on transient wave-propagation problems in the field of elastodynamics and electromagnetics. His current main research interest is the computation of inverse-wave field problems employing iterative techniques based on error minimization.

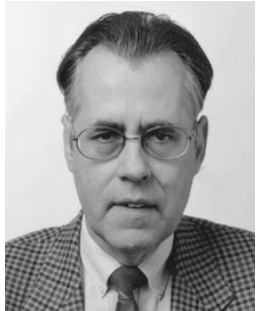

Peter M. van den Berg was born in Rotterdam, The Netherlands, on November 11, 1943. He received the degree in electrical engineering from the Polytechnical School of Rotterdam, Rotterdam, The Netherlands, in 1964, and the B.Sc. and M.Sc. degrees in electrical engineering, and the Ph.D. degree in technical sciences from the Delft University of Technology, Delft, The Netherlands, in 1966, 1968, and 1971 , respectively.

From 1967 to 1968, he was a Research Engineer in the Dutch Patent Office. Since 1968, he has been a member of the Scientific Staff of the Electromagnetic Research Group, Delft University of Technology, where he carried out research and taught classes in the area of wave propagation and scattering problems. During the 1973-1974 academic year, he was Visiting Lecturer in the Department of Mathematics, University of Dundee, Scotland. During a three-month period in 1980-1981, he was a Visiting Scientist at the Institute of Theoretical Physics, Göteborg, Sweden. In 1981, he became a Professor at the Delft University of Technology. From 1988 to 1994, he also carried out research at the Center of Mathematics of Waves, University of Delaware. During the summers of 1993-1995, he was a Visiting Scientist at Shell Research B.V., Rijswijk, The Netherlands. Since 1994, he has also been a Professor in the Delft Research School Centre of Technical Geoscience, Delft, The Netherlands. His current main research interest is the efficient computation of field problems using iterative techniques based on error minimization, the computation of fields in strongly inhomogeneous media, and the use of wave phenomena in seismic data processing. His major interest is in an efficient solution of the nonlinear inverse scattering problem.

Dr. van den Berg was the recipient of the Niels Stensen Stichting Award and a NATO Award. 